\title{
Anticipatory mechanisms and techniques in Bulgarian and British ads and their applications in the English language classroom
}

\author{
Rumyana Todorova*, Zlatko Todorov**
}

\begin{abstract}
The paper deals with various anticipatory mechanisms and techniques used in Bulgarian and British ads and their applications in the English language classroom as ads and commercials are a rich source of different structures and information related to certain cultural and social norms, models and images of behaviour, values and beliefs. Some suggestions for activities with these texts are presented in support of the issues discussed.
\end{abstract}

Key words: anticipatory mechanisms, techniques, advertisements, ELT

\section{Introduction}

Advertising as a type of media discourse abounds in all sorts of topics and constructions suitable for work in class. Advertisements are especially prolific for culture specific discussions as they create, transform and impose familiar models of the world and images not only on the society they originate from, but on other identical, similar, or at times, even different societies or communities, and also presuppose the branding of those models, images and situations which at a later stage can be easily recognized. As for the foreign language classroom, they serve as texts in which the respective values, beliefs and norms of behaviour are reflected. All this enables the learner to better grasp and understand the foreign language culture and traditions which are inevitably part of the reality and environment people live in. Although students may pretend not to be interested in them, ads represent young people's lives so they may comment on them quite actively in the foreign language which is the object of attention in the curricula. As commercials are particularly dynamic and lively young people can follow them and understand them without any difficulties because they represent their way of life, their hobbies and interests, though in a somehow transformed manner. There are cases, however, in which these texts impose certain models of behaviour of young people. On the contrary, many elderly audiences may not even follow them as the information is multilayered in both the verbal and the non-verbal component. The latter is the predominant one as it is more interesting and tempting, although the message is quite schematic and

\footnotetext{
* University of Shumen, Department of English Studies, Shumen, Bulgaria ** University of Shumen, Department of English Studies, Shumen, Bulgaria
} 
fragmented, and it runs very quickly. Yet, it can be decoded by learners quite successfully as they are used to this kind of rendering and grasping information with the help of images which is something near their virtual reality through playing games, watching cartoons, etc.

The information abounds in a number of sociocultural phenomena, valid for the respective period in which advertisements appear, and although it is considered by some that these messages degrade and demoralize young people and spoil their spiritual values, they teach them the lessons of life, in most cases only the positive ones. There are moments in them, however, which imitate quite successfully their actions and reactions (positive or negative) against the background of their favourite music and lyrics, which helps attracting adolescents' attention. This is one of the many ways for remembering foreign words and phrases in real life usages without external imposition as positive feelings and emotions are the predominant ones. These structures not only facilitate easy perception but they enable fulfilling the necessary links between the two components. Thus, language and images can be interconnected on the subconscious level in the direction of cultural codes, aspects and models, normative rules and folklore traditions and customs of the respective culture.

\section{Analysis \& Discussion}

An example of this type of interconnectedness is the commercial about white cheese "Bulgarea" in which banitza is advertised as one of the typical Bulgarian meals (see VBox7_Bulgarea). The Bulgarian tradition of grannies helping with the housework as they live together with their children's families is also brought to the fore as in the ad about mincemeat "Naroden" (folkloric) (see VBox7_Naroden; also Todorova 2014). Thus, family values are exploited and made salient which is something rooted in Bulgarian culture but not discussed that often with young people, especially in connection with their interrelations and attitudes between the different generations. In a similar way, we can ask students to find some other advertisements containing culture specific symbols and issues related not only to Bulgarian but to British culture as well. In the latter case, they are supposed to have been exposed to this culture as they might have read some information from the Internet or from books or might have seen some films containing prompts about it. Teachers can also give them some hints or guiding questions which can orient them in the depicted situation and make them think about certain things they do in their everyday life but they do not always pay attention to them. We can ask them to make comparisons between the two cultures or find some information on the Internet if they are not aware or sure of the answers. For example, a lot of adverts portray young people having fun and enjoying themselves over their mobiles which are of the latest developments in this field. Most probably teenagers are attracted by the phones rather than by the clothes these young people wear. The conversation can be 
focused not only on the brands and some of the stories about the celebrities or the ordinary people promoting the brand products. There can also be questions about these people's lifestyles and behaviour, what learners know about them, what they like or dislike in them, and so on and so forth. They can be asked to compare British and Bulgarian celebrities as representatives of the respective cultures and express their social attitudes towards people in the entertainment industry in general. They may be asked to pay attention to the clothes and shoes the people depicted wear and if they like them or not; and if yes, why they imitate their clothing in some way or another.

As advertisements are a reflection of what happens in society, they can draw young people's attention to the changes and new and recent developments in most spheres in life in a globalizing and highly industrialized world. All abovementioned factors come to prove the possibilities of ad usages in the foreign language classroom.

In this respect, as these texts are quite unpredictable in terms of structure, composition and way of presenting information, they can be exploited in the best way for writing or finishing stories or commenting on the situations presented.

Some anticipatory mechanisms can be offered for the prediction of information. This means that the teacher in question can direct students' attention to places in the text in which there are some prompts related to what comes next or to the advertised object. Such a case is the film "Do-It-Yourself Mr. Bean" (1993) featuring Rowan Atkinson in which Mr. Bean is first buying a lot of reduced items from a department store taking advantage of the January sales, Dulux paint being one of them, and then he is painting his rooms with it though it is not explicitly mentioned. You can see the brand name only sporadically within a fraction of a second. This kind of indirect advertising is a good exercise for students to pay close attention to detail so that they can answer the question about the product used in the film. Nowadays, things have changed and there exists an obligatory statement at the beginning of a film or show in cases in which there is product positioning as a form of marketing technique. So, this is just an example to show that watching a film or any other kind of video material is not done for the sake of enjoyment but it has educational purposes first and foremost as the teacher is the one who will decide what material to play or show to students depending on their level of the foreign language, on the lexical and grammatical units as well as on the cultural aspects that are to be taught or revised.

In another episode of the same film, Mr. Bean puts a sleeping bag with a balloon faking there is a person in it in front of the department store and when time for opening the store comes he jumps in front of the whole queue and pops the balloon happily showing everybody that he has cheated on them. There can be a lot of discussions related to his behaviour in which personal opinions 
can be expressed as well as some presuppositions about what has happened next. Attention is drawn to a number of social and extralinguistic factors which can be described by some words and phrases related to Mr. Beans' actions, movements and reactions. The questions can be: What would you do if you were in the queue? Would you complain publicly or share your indignation with the people around you but not do anything to either block Mr. Bean's way to the entrance or do something else? Can you describe such a person? Students are also asked to identify the communicative strategies and the stereotypes used in the film. Another question related to the last one is if there are stereotypes at all, especially nowadays with the globalization of the world and the unification and elimination of cultural codes.

In connection with the advertisements and commercials that are chosen for discussion and description the method that can be used can be Critical Discourse Analysis in which all the social and cultural discursive practices should be taken into consideration as well as their linguistic manifestations. There is no need for students to know what method is used but it is for facilitators to be aware of this. In cases of commercials the nonverbal or the visual component is the prevalent one and takes precedence. Once it is played, its sound part may be neglected at all or at least some of its parts. What matters is the preliminary conversation related to either the same topics that are to be approached or similar ones. In this way students will be provoked to elicit information referring to their background and previous knowledge about the issues in question as well the thesaurus needed for rendering this knowledge. If they provide words and phrases which coincide with the ones in the narrative, they can be presented other expressions, words and phrases as clues for the commercial, or, vice versa, if they use words and phrases different from the ones in the respective ad messages, then the ones that appear in them, can be put down on the white board for their utilization.

Such texts presuppose positive reactions as they somehow correspond to young people's behaviour, way of thinking, attitudes and lifestyles. It means that students will be willing to work on them irrespective of the fact that some commercials may be familiar to them or others may be quite new to them.

One of the activities connected to the anticipatory mechanisms when approaching these commercials can be playing the nonverbal component (pictures and music) without the narrative so that the possible words and phrases can be elicited on the basis of the predictions made while watching the video clip. Presuppositions can be made about the story and they may turn out to be completely different from the real narrative.

Another activity can be asking students to write a short story related to what they have seen by using their imagination. A good idea would be for the product not to be shown so that they can make guesses what the ad promotes. 
All these tasks will lead to a lot of oral expressions of students' ideas thus making them talk more than usual, which is something they need, as emphasis is usually put on the receptive skills, reading and listening, rather than on one of the productive skills, i.e. speaking, as writing skills are developed more than the former ones.

Activities that are considered easy, but actually they turn out to be quite difficult as the way information is presented seems to be quite misleading at times, are multiple choice questions or a text with omitted words and phrases in the form of cloze tests or even filling in tables with information elicited from the text. No doubt all tasks should be structured according to the corresponding students' level of English.

As for university students, these anticipatory mechanisms can be oriented towards note-taking, which is a skill they lack in most cases, and it is a good exercise for Practical English Classes, but the knack for doing it would be helpful for other subjects as well.

For non-native speakers of English, it is not so easy to differentiate between registers. In most cases the information in ads addressed to youngsters is rendered by words and phrases of a more conversational and colloquial nature, so students can be asked to either compare them with the same words and phrases from the official register or transform the text into this register.

Another difficult activity is related to the way metaphors are interpreted. If they are original metaphors, it is not a problem, but some metaphors may not be familiar to students and they may get them wrong because they may not be able to map the information from the source domain to the target domain correctly. In principle, our minds are structured in such a way that we understand the literal meaning first and then the intended one conveyed in the text. What can also influence our understanding of the information is that there is interaction between the literal text and the text receiver's background knowledge (see Johnson-Laird 1983). This means that students should be asked to "interpret" the literal meaning first and then we can proceed with the metaphorical one. The latter is activated because of our existing global knowledge patterns. On the basis of these links a mental model is built and some inferences are generated thus filling in coherence gaps in text comprehension (see Kintsch 1998), at the same time resolving inconsistencies and incorrect inferences as the text progresses and through the process of decoding the narrative. Comprehension can be blocked not because of the literal meaning but because at first hearing of the message there can be some incongruences which can act as constraints to comprehension.

It is quite difficult for most people to describe a person as regards their appearances and their character as well as their actions. Without playing the narrative students can be asked to perform this activity. A very good example in 
this respect is the Nespresso commercial with George Clooney featuring where the play on words is unmistakable and perfectly exploited. The commercial is full of inconsistencies. It goes in the following way:

George Clooney (acting himself) enters an office in which there are Nespresso machines. On his way in, a lady takes him for a porter and hands him in the keys for her car, saying: "The red one". He is wondering what is happening but puts the keys in his pocket and heads for the coffee machines. A girl stands after him and when she starts talking to him he thinks that she will ask for an autograph, but he is wrong. She is simply asking him to move aside so that she can make herself some coffee. But as she starts saying something, he interrupts her:

'The red one'.

'Excuse me'.

'I'm sorry. Do you mind if I...' (We can ask students to finish the question)

'Of course. You know. I don’t think I have a pen.' (We can ask them what they think about his reactions)

'I just wanted Nespresso'

'Nespresso. What else?

The next moment the lady with the keys comes in and says:

'Excuse me. Where have you been? I've been waiting for my car for about five minutes.'

Clooney thinks she is talking to him as he is famous and everybody knows him, but he is disappointed as she is actually addressing the girl standing next to him:

'Oh, I didn't recognize you...'

'Do you want a...'

'Nespresso. What else?'

(Nespresso1)

The two referential frames are constantly juxtaposed and interwoven.

In another commercial about Nespresso featuring George Clooney again girls are exchanging ambiguous remarks. There comes a moment, however, in which even they do not know if they are talking about one and the same thing (the coffee or Clooney):

'Women:

Dark, very intense, balanced, unique, mysterious... An intense body, delicate and smooth, with strong character... Right. Rich. Very rich. I would say Latin American temperament. Deep and sensual. And a delicious aftertaste.

George Clooney:

You're talking about... Nespresso, right? 
Nespresso. What else?'

(Nespresso2)

The last couple of very short remarks clarifies things and everything is finally made concrete and explicated.

For students with a higher level of English, tasks can even be related to writing a script of the potential narrative before watching the whole commercial.

An interesting activity with some anticipatory tasks and discussions can be paying attention to some extralinguistic aspects in commercials, such as proximity and distance parameters, something culture specific and not easy to detect, especially in cases in which even we as mentors might have not paid attention to or not know.

\section{Conclusion}

To sum up, we can say that advertisements are a rich source of information full of a number of predictions used as anticipatory mechanisms and techniques for the construction of all sorts of foreign language activities on the basis of which students are exposed to simulated real life situations leading to real life reactions on their part not prepared in advance but invented on the spur of the moment, thus practicing the foreign language as much as they can. Ad related activities are highly motivating at that as they bring pleasure, satisfaction and enjoyment - something that is not always present in our everyday life.

\section{References:}

Johnson-Laird 1983. Johnson-Laird P. Mental models: Towards a Cognitive Science of Language, Inference and Consciousness. Harvard: HUP, 1983.

Kintsch 1998. Kintsch W. Comprehension: A Paradigm for Cognition. Cambridge: CUP, 1998.

Nespresso1. $<$ https://www.youtube.com/watch?feature=player embedded $\& v=A x 1 Z 61 \operatorname{RgTDg} \# \mathrm{t}=0>$. Accessed October 30, 2016.

Nespresso2. $<$ http://self-learning-eoicoruna.blogspot.bg/2009/05/george-clooney-whatelse.html>. Accessed October 30, 2016.

Todorova 2014. Todorova R. Ezik, kultura, reklama. Shumen: Konstantin Preslavski Publ. Press, 2014.

Tomlinson 2007. Tomlinson J. "Cultural Globalization". In Ritzer G. (ed.). The Blackwell Companion to Globalization. Malden, MA, USA, Oxford, UK, Carlton, Victoria, Australia: Blackwell Publ. Ltd, 2007. 352-366.

VBox7_Bulgarea. <http://vbox7.com/play:ad6aeb7d71>. Accessed October 5, 2016.

VBox7_Naroden. <http://vbox7.com/play:mdea43a529>. Accessed October 5, 2016. 